\title{
Relaxation technique training to alleviate emotional stress in patients with chronic pain: A report of two cases
}

\author{
Shinobu Kobayashi*, Natsuko Yanagi, Kikuyo Koitabashi \\ Department of Nursing, Gunma University Graduate School of Health Sciences, Maebashi, Japan \\ Email: *kobashino831@gmail.com
}

Received 23 January 2013; revised 26 February 2013; accepted 3 March 2013

\begin{abstract}
Aim: Chronic pain can leads to uncomfortable sensory and emotional experiences and remarkably decrease one's quality of life. The purpose of this report is to describe our experience of treatment with relaxation techniques for two outpatients with chronic pain-related emotional stress. Methods: We offer outpatient relaxation treatment as a specialized outpatient nursing service. Two patients were motivated to self-manage their pain, and thus sought outpatient instruction in relaxation techniques to provide methods of pain self-management. We examined the usefulness of relaxation techniques as pain self-management methods based on subjective information and the Mental Health Pattern questionnaire. Results: Two patients were able to incorporate the relaxation techniques into their daily schedules and control their symptoms. Ultimately, their attitudes toward their pain changed, which lessened their stress levels and improved their quality of life. Conclusions: Our results suggest that nursing care using continuous relaxation techniques improves pain self-management in patients with chronic pain.
\end{abstract}

Keywords: Chronic Pain; Relaxation Technique; Emotional Stress; Self-Management; Specialized Outpatient Nursing Services

\section{INTRODUCTION}

Chronic pain often leads to problems in physical functioning. However, the complex interrelationship of psychological and social factors can increase the severity, extent, and duration of pain [1]. In addition to early treatment to prevent progression to chronic pain, patients should receive comprehensive management involving multiple perspectives, not just pharmacologic treatment $[2,3]$. A recent study reported that $44 \%$ of primary care patients with chronic pain receiving opioid therapy had

\footnotetext{
*Corresponding author.
}

used the complementary and alternative medicine (CAM) in the last 12 months [4].

Relaxation techniques are one of CAM. Practice guidelines for chronic pain management agree that Relaxation techniques should be performed for chronic pain conditions [5]. The recent literature provides evidence for the efficacy of relaxation techniques for a variety of physical (included pain) and psychobehavioral outcomes [6,7].

Our outpatient relaxation clinic offers specialized outpatient nursing services, including training in relaxation techniques for patients with mental and physical health problems. Some of the patients who visit our clinic are obviously under emotional stress, with tension in both their minds and bodies due to anxiety associated with chronic pain. This report describes two patients with chronic pain who were taught relaxation techniques to increase their perceived pain self-management.

\section{METHOD}

\subsection{Subjects}

Two patients with chronic pain receiving relaxation techniques from our outpatient relaxation clinic services were recruited. Each physician referred a patient to our clinic. The inclusion criteria included being 20 years old or above and able to read, write and currently receiving relaxation techniques for their pain self-management [8].

The personal information of both patients has been deidentified, and an in identifier number prescribed. They gave consent for the publication of personal health information in print (without divulging personal identifiers).

\subsection{Outline of Outpatient Relaxation Service}

We offer outpatient relaxation treatment in the form of weekly group training. Relaxation techniques are taught as a method of self-management. During the first session, a breathing method is taught, which provides the basis of the relaxation techniques. We also teach progressive muscle relaxation, autogenic training and guided imagery. We recommend that patients try to attend the weekly 
sessions for approximately three months (a total of 12 visits) and that they practice the techniques at home on the days they do not visit our clinic.

The Mental Health Pattern questionnaire (MHP-1) [9] is administered to our patients approximately once a month to evaluate changes in their mental health during treatment. The MHP-1 includes a Stress Check List (SCL), which yields an overall stress score, and an assessment of quality of life (QOL). SCL scores range from 30 to 120, with higher scores indicating greater stress. QOL scores range from 10 to 40, with higher scores indicating greater QOL. The MHP-1 is a psychometrically validated instrument with high internal consistency (Cronbach's Alpha of SCL $=0.911$, Cronbach's Alpha of QOL = 0.835) [9].

\section{CASE REPORTS}

\subsection{Case 1: Female, 50s}

The chief concern in Case 1 was chronic pain extending from the patient's lower abdomen to both calves, with occasional upper body pain. The patient had undergone surgical treatment of ileus 2 years prior to enrollment in our clinic. She sought treatment of her pain and complained of anxiety related to her illness and pain. The patient expressed her desire to self-manage pain to the highest possible extent.

She lived with her husband and two children, and her husband was always attentive and supportive.

\subsubsection{History of the Condition}

The pain commenced approximately 1 to 2 months after the patient had undergone surgical treatment of ileus. The pain was localized in her lower body. The patient later received pharmacologic treatment, but the symptoms did not improve. The idiopathic pain caused anxiety, which in turn led to insomnia. When it became obvious that the patient's emotional and physical stress levels were increasing, her attending physician referred to our clinic for outpatient relaxation treatment.

\subsubsection{Process}

After the first training session, the patient stated that the patient found the breathing technique "difficult". She was instructed not to be overly concerned about doing the exercise correctly. Initially, she was unable to complete the exercises because she was afraid of experiencing pain. The patient had persistent pain almost every morning. She was willing to persevere with the exercises, and stated, "It won't get better right away, but I'd like to continue at my own pace, little by little”. At the third training session, the patient was able to attain feelings of relaxation and heaviness in her body. The patient reported that she was able to take her mind off the pain during the daily practice. We advised the patient that the relaxation technique would not have the same quick results as pharmacologic treatment, but that it was important to continue paying attention to the gradual changes that were occurring in the body and mind.

Approximately one month later, the patient's facial expression was brighter, and she was more talkative. The MHP-1 scores were markedly improved (Figure 1). She felt that her stress level was gradually decreasing.

The patient continued to practice the relaxation techniques whenever she felt increased the pain or anxiety. Referring to the positive results, she said, "It may only be slight, but I've become more positive in my way of thinking about things", and, "I'm better able to sleep without using sleep medications".

Approximately two months after the first session, the patient was cheerful and talkative with other patients. She continued the daily practice whenever the pain would appear and at bedtime. "I sometimes wake up at night with pain all over my body. I don't panic. I just practice the breathing method, and fall back asleep. In the morning, I practice the relaxation technique in bed. That way, I can alleviate some of the tension and get up with less pain". According to the patient, she had begun to self-manage the pain and sleep better by using the relaxation techniques. The patient said that she was more cheerful and relaxed about the pain. She also experienced changes in her attitude toward the pain.

These first two months were a time of gradual, progressive improvement. We advised her that in her daily life, there would be improvements as well as setbacks. It was important that the patient did not worry about them. Rather, the patient should accept that "there will be times like these", and then let them go.

After that, the patient began to attend training sessions less frequently, but she continued to practice the relaxation techniques almost every day, and was better able to

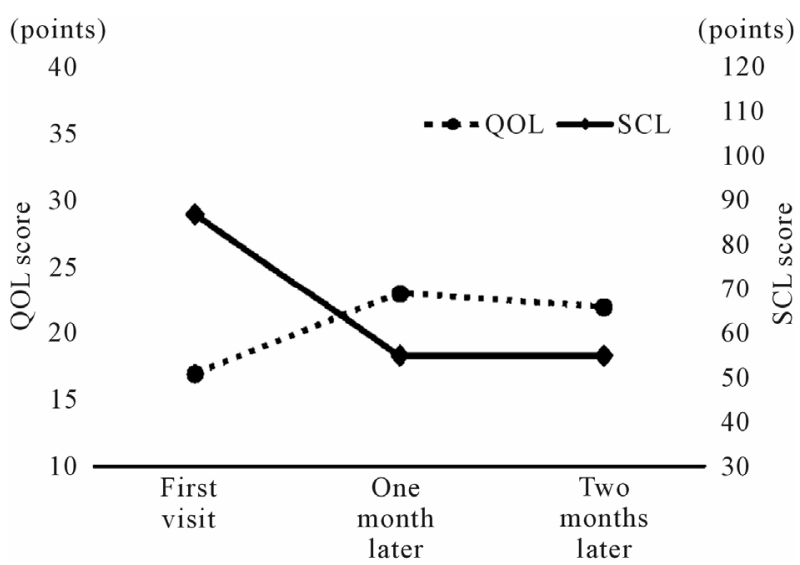

Figure 1. Case 1 (Female, 50s), change in MHP-1 scores. The chief concern was chronic pain extending from the patient's lower abdomen to both calves. 
integrate these techniques into her daily life. The patient did not feel that the severity or frequency of the pain had changed, but she had become better able to deal with it. By integrating the relaxation techniques into her life, the patient clearly changed the attitude toward her pain.

\subsection{Case 2: Male, 30s}

The chief concern in Case 2 was pain and discomfort on the left side of the patient's face that was caused by trigeminal neuralgia. The pain sometimes extended over a wide area, and the intensity was irregular. The patient had bronchial asthma. He wished to learn a stress-reduction technique. His physician had told him that the pain resulted from stress, but he did not know the cause of the stress.

He was office worker and on the regular payroll. He lived with his parents.

\subsubsection{History of the Condition}

Four years previously, the patient had presented with discomfort and pain on the left side of his face, which was diagnosed as trigeminal neuralgia. Subsequent medical treatment had little effect on the symptoms. The extent and severity of the pain were irregular, although he sometimes found it difficult to walk when the pain was particularly severe. The physician's suggestion that stress might increase the severity of the pain motivated the patient to visit our outpatient relaxation service to learn stress-reduction techniques.

\subsubsection{Process}

The first training session was the patient's first experience with relaxation techniques. However, the patient was able to comfortably follow the instructions. We asked him for details about the frequency and extent of the pain and tried to develop ways he could use the relaxation technique within the context of the daily life. At the second session (two weeks later), the patient practiced the progressive muscle relaxation technique. He found that technique more difficult because of the need for physical movement. He stated that he was practicing the breathing method daily but had not felt any effect. We told him that the effects would not appear immediately and that he should continue practicing as often as possible.

At the third session (three weeks later), during the training, the patient experienced uncomfortable prickling sensations extending from his cheeks to the corner of mouth, as well as facial numbness. He began to think, "try to make it go away" and was unable to concentrate on the training.

The patient had been continuing the breathing practice technique since the previous visit, but pain symptoms appeared very frequently and he felt unable to control them. Because the symptoms were affecting his work, his inability to control their appearance was leading to substantial emotional stress. We discussed strategies for addressing the symptoms, using relaxation techniques. We told him that the patient could expect improvement in the symptoms even at work, if he simply repeated the breathing techniques a few times

Approximately one month later, the patient did not mention any feelings of relaxation or improvement of the symptoms. In fact, his MHP-1 scores were little changed (Figure 2). In addition to providing instruction on the relaxation techniques, we listened carefully to his concerns and reassured him that substantial changes in his way of approaching and feeling pain were possible.

Approximately two months after first session, the patient said that he felt good, and he had a cheerful expression. After reflecting on his daily improvements, he continued to practice relaxation techniques until the pain disappeared. Until recently, the patient had been tensing up in response to the pain, but he said that he now understood that he could better avoid pain by relaxing and releasing tension, which indicated that he was beginning to find ways to use his training in the daily life.

We told the patient that when practicing the techniques during daily life, it was acceptable to only use parts of the techniques. The patient was now able to use the techniques to lessen pain whenever the uncomfortable prickling sensation appeared. He repeated the relaxation technique at work and at bedtime. This resulted in a feeling of refreshment and he was increasingly able to avoid the symptoms. As a result, the patient felt "reduced irritation and stress caused by pain by learning these relaxation techniques”.

\section{DISCUSSION}

The treatment of chronic pain now includes both non-

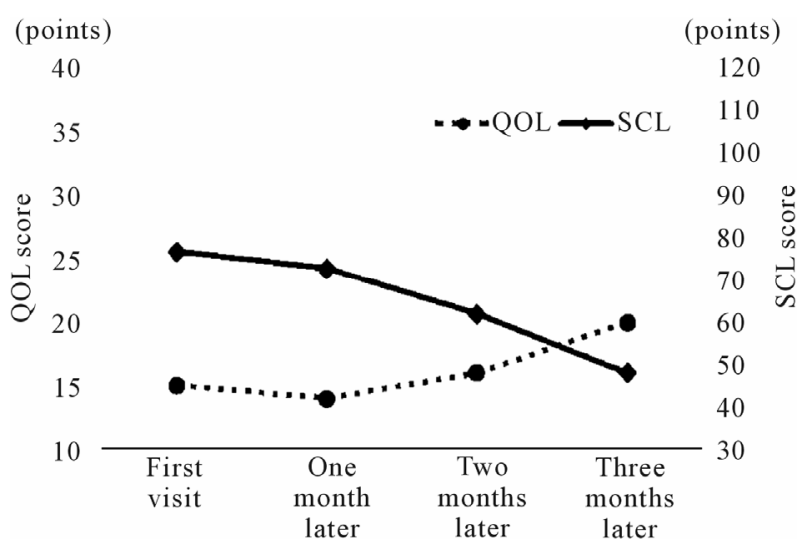

Figure 2. Case 2 (Male, 30s), change in MHP-1 scores. The chief concern was pain and discomfort on the patient's face that was caused by trigeminal neuralgia. 
pharmacologic and pharmacologic methods. The guidelines for the treatment of Chronic Pain $[3,5,10]$ recommend the use of cognitive-behavioral therapy and relaxation techniques. In recent years, relaxation techniques have been taught in nursing education programs and are now seen as a distinct care modality provided only by nurses. In the present two cases, continued nurse-administered training in relaxation techniques supported them to learn pain self-management.

At first presentation to our training sessions, the patients were unable to immediately relax or alleviate their pain, and complained about the difficulty of the techniques. We listened carefully to the patients' expressions of pain and anxiety and responded in accordance with these expressed needs. In addition, to facilitate mind and body relaxation, we conducted the training in a relaxed and laid-back environment.

After a month, the patients began to notice effects and changes. Case 1 reported being able to sleep better and an improvement in the thinking patterns, i.e., she had become more positive in the outlook. In the early stage, her awareness of mind and body should increase, and after that, it would be easier to reflect on pain and emotional stress. However, like Case 2, the patient's mental state remained somewhat unsettled. His pain symptoms had not improved. In this stage, he continued to be affected by his pain. He could not feel a sense of self-control. Since he led a busy life, and was of a young and productive age, he felt difficulties having time to practice relaxation techniques.

Educating patients about relaxation techniques, and encouraging them to continue these practices, is extremely important in supporting patients with pain. By carefully listening to the timing and location of pain, we can plan how and when to use relaxation techniques. Similar to what we experienced from the two cases, the arrangements of relaxation techniques will be necessary to consider the following factors: lifestyle, personality, age and sex $[6,11,12]$. An added benefit of such careful dialogue was that the patients became more aware of their conditions. They were therefore better able to decide suitable times and places for relaxation techniques and acquired the ability to avoid experiencing painful symptoms. We found that this new ability greatly increased their confidence, helped reduce their levels of anxiety and emotional stress, and aided them to acquire a sense of self-control. The MHP-1 scores confirmed these improvements. Rosenzweig et al. [13] and Chen and Francis [6] also found that relaxation techniques improved QOL and mental health in patients with chronic pain. Even with traditional pain treatment methods, patients with chronic pain sometimes experience an alleviation of their symptoms when they come to accept the pain. It has therefore been suggested that the goal should not be the complete eradication of pain. Instead, the focus should be on the patient's attitude toward the pain, i.e., the acceptance of it.

\section{STUDY LIMITATION}

This report is a case study, which is a pragmatic first step in researching a nursing care. Conversely, a major limitation to such research reports is a lack of generalizability.

In the present two cases had been wanted to care by themselves from the beginning of the treatment. They had a high motivation to treatments. Earlier research has shown that patients' attitudes toward treatment significantly affect treatment outcomes [14]. Thus further longterm prospective cohort studies are required to establish the significance of effects of relaxation techniques for chronic pain. And further research will focus on the systematic evaluation of personality and psychological factors.

\section{CONCLUSION}

Findings suggest that for these cases, providing instruction on relaxation techniques to patients with chronic pain was useful in helping them achieve self-control. Support for patients with chronic pain requires a psychological approach that encourages long-term treatment in addition to life counseling to help alleviate stress-an approach derived from a model of personal growth. We hope that more nurses will actively promote the benefits of relaxation techniques for patients with chronic pain.

\section{REFERENCES}

[1] Dailey, P.A., Bishop, D.G., Russell, I.J. and Fletcher, E.M. (1990) Psychological stress and the fibrosists/fibromyalgia syndrome. The Journal of Rheumatology, 17, 1380-1385.

[2] Japan Ministry of Health, Labour and Welfare (2010) Round table on chronic pain: Strategies for dealing with chronic pain from now on (statement).

http://www.mhlw.go.jp/stf/houdou/2r9852000000ro8f-att /2r9852000000roas.pdf

[3] Adopted in WHO Steering Group on Pain Guidelines (2008) Scoping document for WHO treatment guideline on non-malignant pain in adults.

[4] Frank, H.L. and Srinivasa, N.R. (2011) Complementary and alternative medicine in chronic pain. PAIN, 152, 2830. doi:10.1016/j.pain.2010.09.023

[5] Practice Guidelines for Chronic Pain Management (2010) Anesthesiology, 112, 1-24. doi:10.1097/ALN.0b013e3181c5f88d

[6] Chen, Y.L. and Francis, A.J. (2010) Relaxation and imagery for chronic, nonmalignant pain: Effects on pain symptoms, quality of life, and mental health. Pain Management Nursing, 11, 159-168. 
doi:10.1016/j.pmn.2009.05.005

[7] Baird, C.L. and Sands, L. (2004) A pilot study of the effectiveness of guided imagery with progressive muscle relaxation to reduce chronic pain and mobility difficulties of osteoarthritis. Pain Management Nursing, 5, 97-104. doi:10.1016/j.pmn.2004.01.003

[8] Bonnie, L.K., Karen, S.D. and Mark, R.S. (2009) Daily activity patterns of an adult experiencing lower back pain undergoing electro-acupuncture: A case study. Pain Management Nursing, 10, 188-196. doi:10.1016/j.pmn.2008.06.001

[9] Hashimoto, K. and Tokunaga, M. (2000) Mental health patterns: A diagnostic inspection manual. Toyo Physical Co., Fukuoka.

[10] Tsuji, S., Ushida, T., Arai, K., Suetomi, K., Nishihara, M., Ikemoto, T., et al. (2010) Guidelines for chronic painstandard neurological treatment-neurological therapeutics. Japanese Society of Neurological Therapeutics, 27, 591622.
[11] James, J.K., Tricia, A.M., Andrew, J.M. and Charles, R.R. (2008) Complementary/alternative medicine use among chronic pain clinic patients. Journal of PeriAnesthesia Nursing, 23, 17-23. doi:10.1016/j.jopan.2007.05.003

[12] Anna-leila, W., Peter, V.N., Jane, D. and Ruth, M. (2012) Barriers to meditation by gender and age among cancer family caregivers. Nursing Research, 61, 22-27. doi:10.1097/NNR.0b013e3182337f4d

[13] Rosenzweig, S., Greeson, J.M., Reibel, D.K., Green, J.S., Jasser, S.A. and Beasley, D. (2010) Mindfulness-based stress reduction for chronic pain conditions: Variation in treatment outcomes and role of home meditation practice. Journal of Psychosomatic Research, 68, 29-36. doi:10.1016/j.jpsychores.2009.03.010

[14] Goossens, M.E., Vlaeyen, J.W., Hidding, A., Kole-Snijders, A. and Evers, S.M. (2005) Treatment expectancy affects the outcome of cognitive-behavioural interventions in chronic pain. Clinical Journal of Pain, 21, 18-26. doi:10.1097/00002508-200501000-00003 